\title{
A MODELLING OF EXPANSION VELOCITIES OF PLANETARY
}

\section{NEBULAE}

\author{
K.GESICKI and R.SZCZERBA \\ Nicolaus Copernicus Astronomical Center, Chopina 12/18, 87-100 Torun, POLAND
}

Recently written model of photoionization structure of planetary nebula has been used to calculate the emissivities of different nebular lines. With these values the line profiles have been modelled. The profiles can be obtained for different size and position of the spectrograph slit on the nebula image. The prepared computer code allows for estimation of the expansion velocities $\left(\mathrm{V}_{\exp }\right)$ in the way consistent with the methods used by observers, i.e. defined as the half-width of the line profile (when nebula is not resolved, "whole neb." in the Figure) or defined as the half of the emission peaks separation (when the nebula can be resolved - "rectangle" in the Figure - and the line can be observed in the central part of the nebula). The real matter velocity at the radius where the observed surface brightness of $\mathrm{H} \beta$ falls below $20 \%$ of the maximal value is shown for comparison ("model" in the Figure).
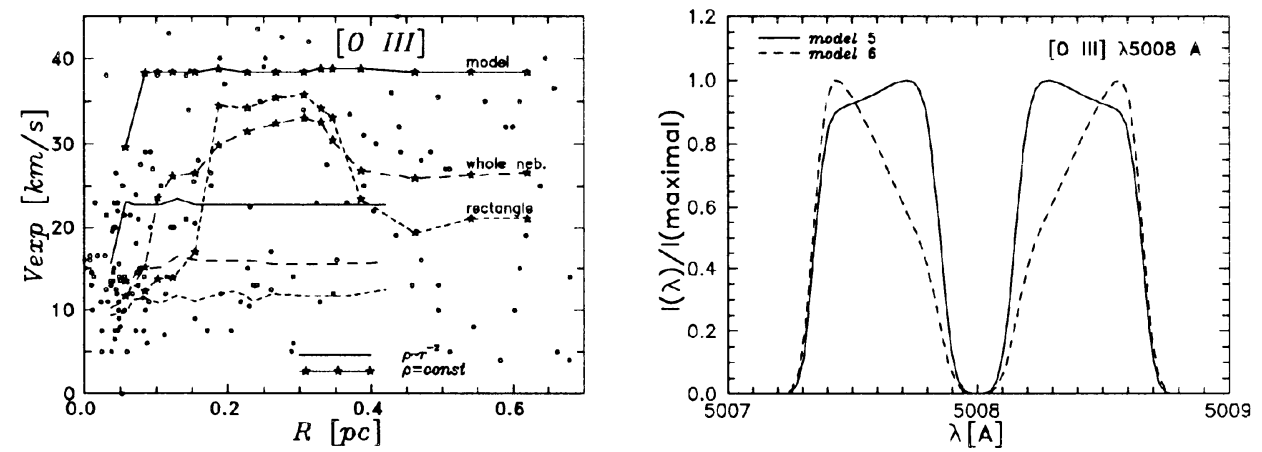

Figure: The obtained evolutionary sequences (left) and an example of calculated profile (right)

The simple models of planetary nebula evolution (shell expanding with $\mathrm{V}_{\text {exp }}$ $=40 \mathrm{~km} / \mathrm{s}$ at the outer radius and $10 \mathrm{~km} / \mathrm{s}$ at the inner edge, when the central star follows Schönberner's model of $0.598 \mathrm{M}_{\odot}$ ) agree quite well with the observed correlations of $\mathrm{V}_{\text {exp }}$ versus planetary radius, but rather do not follow the correlation of $\mathrm{V}_{\text {exp }}$ versus excitation class of nebula as measured by line ratio $\mathrm{I}(\mathrm{HeII}$ $\lambda 4686) / \mathrm{I}(\mathrm{H} \beta)$. The presented line shapes show how they are influenced by ionization structure of the nebula, and movement of the ionization front explains the jump in measured velocities seen in the left Figure for model with constant density distribution. 